\title{
An ordered metallic glass solid solution phase that grows from the melt like a crystal
}

\author{
Karena W. Chapman ${ }_{\lambda}^{a}$, Peter J. Chupas ${ }_{\lambda}^{a}$, Gabrielle G. Long ${ }_{\lambda}^{a, b, *}$, Leonid A. Bendersky ${ }_{\curlywedge}^{b}$,

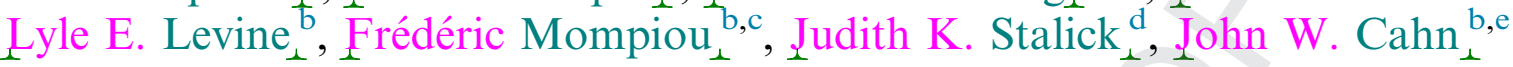 \\ ${ }^{a} X$-Ray Science Division, Argonne National Laboratory, Argonne, IL 60439, USA \\ ${ }^{\mathrm{b}}$ Metallurgy Division, National Institute of Standards and Technology, Gaithersburg, MD 20899, USA \\ ${ }^{\mathrm{c}}$ Centre d'Élaboration de Matériaux et d'Études Structurales, CNRS, 31055 Toulouse, France \\ ${ }^{\mathrm{d}}$ NIST Center for Neutron Research, National Institute of Standards and Technology, Gaithersburg, MD 20899, USA \\ ${ }^{\mathrm{e}}$ Department of Physics and Astronomy, University of Washington, Seattle, WA 98195, USA
}

Received 8 July 2013; received in revised form 29 August 2013; accepted 31 August 2013

Abstract

We report structural studies of an Al-Fe-Si glassy solid that is a solid solution phase in the classical thermodynamic sense. We demonstrate that it is neither a frozen melt nor nanocrystalline. The glass has a well-defined solubility limit and rejects Al during formation from the melt. The pair distribution function of the glass reveals chemical ordering out to at least $12 \AA$ that resembles the ordering within a stable crystalline intermetallic phase of neighboring composition. Under isothermal annealling at $305^{\circ} \mathrm{C}$ the glass first rejects Al, which persists for approximately $1 \mathrm{~h}$ with no detectable change in structure, and finally is transformed by a first-order phase transition to a crystalline phase with a structure that is different from that within the glass. It is possible that this remarkable glass phase has a fully ordered atomic structure that nevertheless possesses no long-range translational symmetry and is isotropic.

(C) 2013 Published by Elsevier Ltd. on behalf of Acta Materialia Inc.

Keywords: Metallic glass; Phase transformation; Pair distribution function analysis; X-ray scattering

\section{Introduction}

Many ordered three-dimensional (3-D) arrangements of points are neither periodic nor quasi-periodic [1], where "order" means that the positions of the points are determined. Physical realizations would be structures that are neither crystalline nor quasi-crystalline. We recently reported initial results on a new isotropic $\mathrm{Al}-\mathrm{Fe}-\mathrm{Si}$ alloy, a metallic glass produced by rapid solidification of the melt, which may possess such a structure [2]. Here, we present the complete experimental results and analyses that support this conclusion.

* Corresponding author at: X-Ray Science Division, Argonne National Laboratory, Argonne, IL 60439, USA. Tel.: +1 3018738365.

E-mail address: gglong@aps.anl.gov (G.G. Long).
Three general concepts are usually applied to glass formation from metallic alloy melts. First, there is the long-established view that glasses are frozen melts. Frozen melt glasses have the same composition as the melt and inherit the local ordering present in the melt at temperatures just above those where dynamic motion ceases. Supporting this concept is a large body of literature describing many glass-forming systems. Second, there is the view that metal alloy glasses are really polycrystalline with nanoscale structural ordering. A third view is that metallic alloy glasses can be composed of low energy, well-defined local atomic structures. A consequence of this third view is the expectation that local atomic arrangements in these glasses closely approximate structures in crystalline phases of similar composition.

These three concepts lead to different predictions about how the glass forms and what the final ordering will be, 
and clearly no single approach describes all glasses. For example, the low temperatures reached by eutectic melts occur because there are no stable crystalline phases at the eutectic composition. Frozen eutectic melts nevertheless are achieved, and this occurs not only because there is a large nucleation barrier, but also because crystal growth is slowed by necessary diffusion [3]. The structure of such glasses would not approximate a crystalline phase.

We here present an example of a metallic alloy glass (the "q-glass") that does not form as a frozen melt. Its formation, quite remarkably, exhibits many characteristics more typical of crystalline phases. In particular, the q-glass is a solid phase that grows from the melt by means of a nucleation and growth mechanism; there is chemical partitioning and atomic rearrangement at the glass-liquid interface in the manner of a crystalline solid. Despite this classical nucleation and growth, this phase is a glass in the sense that it is isotropic (within measurement uncertainty) and exhibits no translational ordering.

The existence of the q-glass raises many questions that we address below. What is the local structure of the q-glass? How does its structure compare with those of crystalline phases of similar composition? Is it a true glass or a nanocrystalline aggregate? How stable is it? When it devitrifies, what phases form? More fundamentally, is it a true metastable phase in a thermodynamic sense?

\section{Morphological character of the q-glass}

We investigated specimens that are 100\% q-glass, or nearly so, examined their formation mechanism, and provide a detailed analysis of the q-glass atomic scale structure to uncover possible similarities to neighboring phases. We also followed the q-glass behavior during isothermal annealing to test whether the q-glass might be nanocrystalline.

The first indication that the q-glass is not an undercooled melt that has become kinetically frozen came from the microstructural morphology of the formation of the q-glass [2]. q-Glass nodules are at the center of radiating patterns of crystallization, indicating that the glass was first to solidify from the melt $[2,4]$. This is in stark contrast to a typical frozen melt glass, which would be the last phase to form. As it grows the q-glass depletes the surrounding melt of iron and silicon, indicating that the q-glass forms from the melt as a primary isotropic phase by nucleation followed by growth. During growth there is a partitioning of the chemical species at the interface between the q-glass and the melt. These factors imply the coexistence of phases, which is a defining feature of a first order phase transition in multicomponent systems. This remarkable behavior was observed in a series of rapid solidification (melt spinning, atomization and e-beam surface melting) studies of the formation of glassy Al-Fe-Si alloy compositions [3,5]. While metallic glasses that are frozen melts appear to tolerate compositional variations as broad as those of the chosen melt compositions, this $\mathrm{Al}-\mathrm{Fe}-\mathrm{Si}$ q-glass behaves instead like a growing crystal, where atomic species that do not fit well into the structure are rejected. Specimens that are $100 \%$ q-glass (Fig. 1a) can only be grown with a composition close to 15 at. $\% \mathrm{Fe}, 20$ at. $\% \mathrm{Si}$, which is close to the compositions of the $\alpha$-cubic Al-Fe-Si phase $[6,7]$ and an icosahedral phase. The $\alpha$-cubic phase is stable in the Al$\mathrm{Mn}-\mathrm{Si}$ system, and metastable in the $\mathrm{Al}-\mathrm{Fe}-\mathrm{Si}$ system. Further, we note that there is no nearby eutectic in the phase diagram [8]. Even near the 100\% q-glass composition evidence for q-glass formation by a moving interface can be seen in Fig. 1b. A trapped gas bubble between the melt and the cooling wheel has slowed glass formation enough that partitioning begins to occur midway through the foil.

\section{Sample preparation and preliminary characterization}

To find concentrations that yield 100\% q-glass specimens of varying alloy composition were prepared from a series of melts subjected to rapid solidification, after which they were characterized by laboratory based X-ray diffraction (XRD), transmission electron microscopy (TEM), and selected area electron diffraction (SAED). The Al-Fe-Si phase diagram showing the liquidus projections [8] is given in Fig. 2. The red region indicates the region of stability for the quasi-crystal plus $\mathrm{Al}$, the yellow region indicates the stable compositional range for the polycrystalline $\alpha$-cubic approximating the quasi-crystal, and the blue region indicates the compositional range where the q-glass plus $\mathrm{Al}$ is stable. The composition of the q-glass specimen that is the subject of this paper is marked with a red star near the $15 / 20$ composition. We note that the $\beta$-phase is designated $\beta-\mathrm{Al}_{4.5} \mathrm{FeSi}$ in Fig. 2, in agreement with both this paper and other papers in the literature, such as Liu and Chang [9]. This phase is sometimes referred to as $\tau_{6}$.

For the research reported here rapidly solidified samples were prepared by melt spinning in a helium environment using a $40 \mathrm{~cm}$ diameter $\mathrm{Cu}$ wheel rotating at 3000 r.p.m. The quenched ribbons were typically 5-10 $\mathrm{mm}$ long, by $5 \mathrm{~mm}$ wide, by $30-50 \mu \mathrm{m}$ thick. Samples were screened by means of XRD and TEM. XRD was used as a primary tool to identify samples that were close to $100 \%$ of the phase of interest. The samples that consisted of nearly $100 \%$ q-glass (g-Al-Fe-Si) had the composition $\mathrm{Al}-15 \mathrm{Fe}-20 \mathrm{Si}$, those of nearly $100 \% \alpha$-cubic $\mathrm{Al}-\mathrm{Fe}-\mathrm{Si}(\alpha-\mathrm{Al}-\mathrm{Fe}-\mathrm{Si})$ had the composition $\mathrm{Al}-18 \mathrm{Fe}-8 \mathrm{Si}$, and the predominantly icosahedral phase (ico-Al-Fe-Si) had the composition Al-18Fe-22Si. TEM and ultra-small angle X-ray scattering (in sector 32-ID at the Advanced Photon Source, Argonne National Laboratory) analyses of the selected samples confirmed the XRD measurements.

\section{High energy X-ray scattering and determination of the static structure}

To probe the short-range and medium-range order of the q-glass we performed high energy X-ray scattering to large values of momentum transfer $Q$. This enables accurate Fourier transformation of the data to recover the pair 
(a)

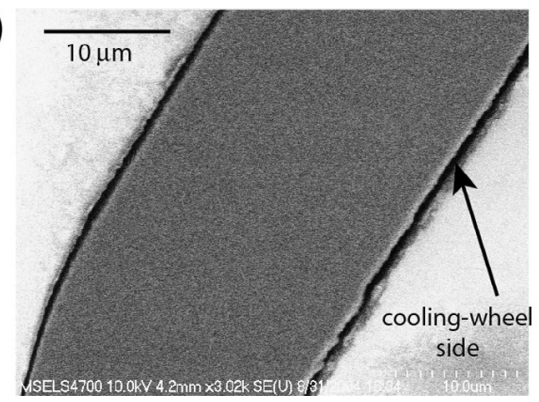

(b)

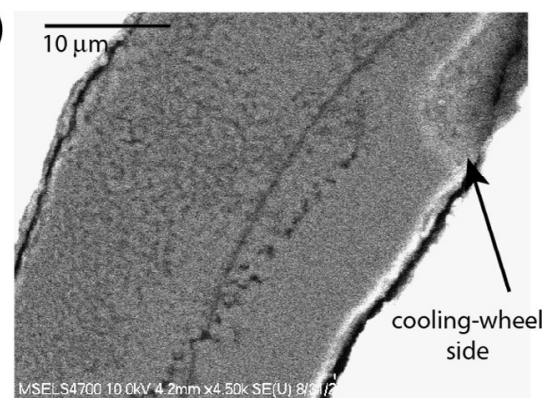

Fig. 1. (a) SEM image of a cross-section of a melt spun ribbon of the $\mathrm{Al}-15 \mathrm{Fe}-20 \mathrm{Si}$ at.\% alloy showing a uniform glassy structure throughout the thickness and (b) SEM image of a cross-section of the same alloy near a bubble which slowed cooling, showing a uniform glassy structure on the cooling wheel (right) side, which develops into a heterogeneous glass $+\mathrm{Al}$ structure to the left of a clearly marked boundary.

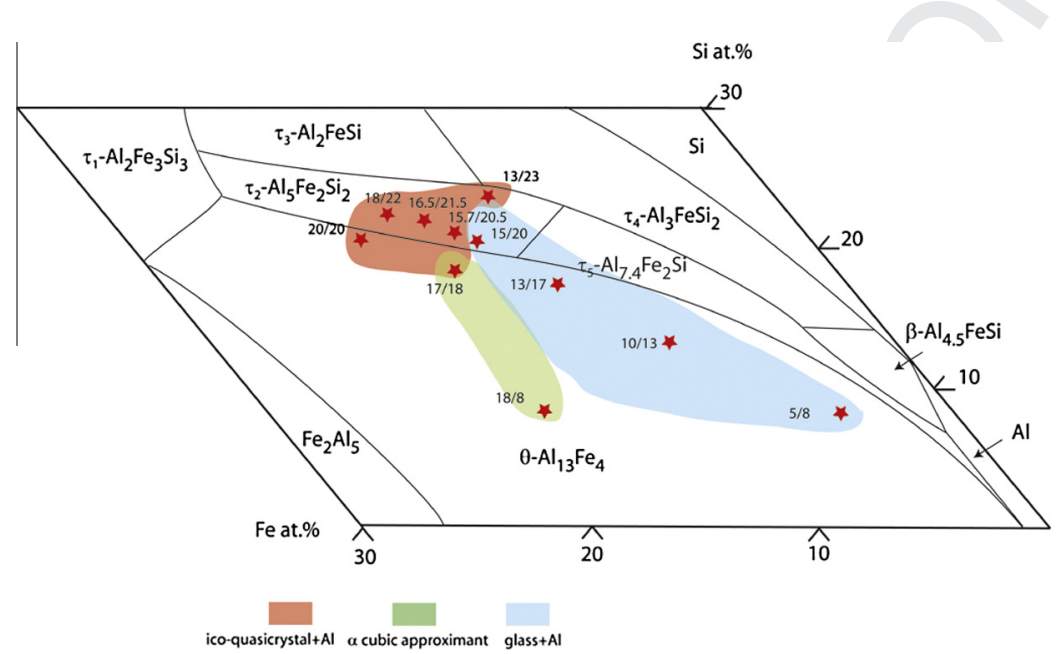

Fig. 2. Al-Fe-Si diagram showing the liquidus projections (after Ghosh [8]). The red region indicates where we find quasi-crystal $+\mathrm{Al}$; the green region indicates where we find the polycrystalline $\alpha$-cubic approximating the quasi-crystal; the blue region indicates where we find the q-glass $+\mathrm{Al}$. The compositions that were studied are marked with red stars. The composition of the q-glass specimen $(15 / 20)$ that is the subject of this paper is marked with the red star near the intersection of the red, green, and blue regions. (For interpretation of the references to color in this figure legend, the reader is referred to the web version of this article.)

distribution function (PDF) [10]. The PDF formalism enables the derivation of structural information in real space in the form of a probability distribution of atomatom correlations. The method is widely used to study the structure of glasses and other disordered materials, where interest typically lies in the first, second and third coordination shells of the structure, and the information extracted usually consists of bond lengths and coordination numbers. Elucidating the nature of $\mathrm{g}-\mathrm{Al}-\mathrm{Fe}-\mathrm{Si}$ requires further identification of the intermediate-range structure, as will be seen in what follows. Fitting complex structural models solely to one-dimensional PDF data can be challenging and often must make use of careful analogies to known crystalline forms. To address this we analyzed the local structure of g-Al-Fe-Si, and investigated its relationship to $\alpha-\mathrm{Al}-\mathrm{Fe}-\mathrm{Si}$ and ico-Al-Fe-Si. The samples are listed in Table 1.

High energy $(80.725 \mathrm{keV}, \lambda=0.15359 \AA)$ X-ray scattering data suitable for PDF analysis were collected in the APS beamline 1-ID-C [11] at the Advanced Photon Source, Argonne National Laboratory, on samples of
$\alpha-\mathrm{Al}-\mathrm{Fe}-\mathrm{Si}$, ico-Al-Fe-Si and g-Al-Fe-Si. Square X-ray beams were defined by means of slits with unidirectional focusing of the beam to enhance the flux at the smaller beam sizes. Small beam sizes, $30 \times 30 \mu \mathrm{m}$, are a good match to the thickness of the samples $(\sim 40 \mu \mathrm{m})$. Each foil-like sample was mounted orthogonally to the beam and step scanned along a square grid with dimensions matching that of the beam size to allow diffraction data to be collected at each point (see Fig. 3). The two-dimensional diffraction images $(2048 \times 2048$ pixels $)$ were measured using an amorphous silicon-based area detector [12] produced by General Electric Healthcare mounted orthogonal to the beam path and centered on the beam. The sample to detector distance and the tilt of the detector relative to the beam were refined using a $\mathrm{LaB}_{6}$ standard within the analysis software Fit-2D [13]. The raw images were corrected for gain, pixel efficiency, bad pixels and dark current. The corrected two-dimensional (2-D) images were integrated within Fit-2D to obtain the onedimensional powder diffraction pattern, masking areas obscured by the beam stop arm [14]. 
Table 1

List of samples and fitted lattice parameters.

\begin{tabular}{llll}
\hline Sample & Phase & Composition (at.\%) & Lattice parameter $a(\AA)$ \\
\hline$\alpha-\mathrm{Al}-\mathrm{Fe}-\mathrm{Si}$ & Crystalline $\alpha$-cubic & $\mathrm{Al}-18 \mathrm{Fe}-8 \mathrm{Si}$ & 12.55 \\
ico-Al-Fe-Si & Quasi-crystalline icosahedral & $\mathrm{Al}-18 \mathrm{Fe}-22 \mathrm{Si}$ & 12.06 \\
g-Al-Fe-Si & q-Glass & Al-15Fe-20Si & 12.26 \\
\hline
\end{tabular}
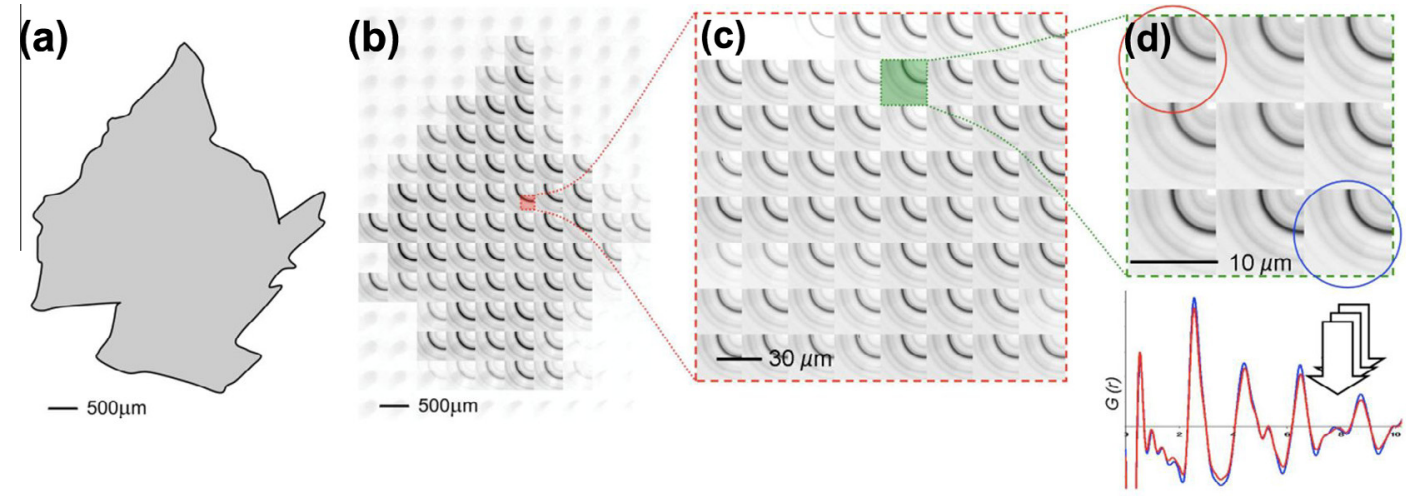

Fig. 3. The spatially resolved diffraction measurements performed on the g-Al-Fe-Si sample. (a) The shape of the g-Al-Fe-Si sample. Spatially resolved measurements using (b) a $500 \times 500 \mu \mathrm{m}$ beam, (c) a $30 \times 30 \mu \mathrm{m}$ beam, and (d) a $10 \times 10 \mu \mathrm{m}$ beam. A quadrant of the 2-D diffraction pattern is shown in each square of the grids. Areas with texture are evident on length scales of $30 \mu \mathrm{m}$. We used the red and blue circled diffraction patterns on the $10 \mu \mathrm{m}$ scale to extract the PDFs. Since these PDFs, shown below the $10 \mu \mathrm{m}$ grid, are equivalent within error they demonstrate the uniformity of the sample on the $10 \mu \mathrm{m}$ length scale. (For interpretation of the references to color in this figure legend, the reader is referred to the web version of this article.)

Preliminary X-ray measurements revealed that the $\alpha$-Al$\mathrm{Fe}-\mathrm{Si}$ and the ico-Al-Fe-Si samples included a degree of diffraction texture (i.e. made visible by the non-uniformity of the diffraction rings) over the $500 \times 500$ and $30 \times 30 \mu \mathrm{m}$ length scales, where these varied across the sample regions examined. The g-Al-Fe-Si sample was not entirely uniform, with isolated inclusions of face-centered cubic (fcc) Al crystallites. Even within apparently uniform regions such as that shown in Fig. 3c there was occasional evidence, in the form of slight diffraction texture, of very fine non-uniformities. To identify the most uniform glassy regions areas of the sample with minimal diffraction texture were selected in the $30 \times 30 \mu \mathrm{m}$ scans and used for the finer scale $10 \times 10 \mu \mathrm{m}$ scans. The spatially resolved mapping allowed us to isolate diffraction patterns from each of the samples that were free of significant texture and/or of single crystal spots, and it enabled us to obtain data suitable for PDF analysis for each of the three phases of interest.

The PDF analysis made use of the diffraction data collected with the smallest $(10 \times 10) \mu \mathrm{m}$ beam. Contributions to the scattering from the sample environment and background were subtracted. Corrections for multiple scattering, $\mathrm{X}$-ray polarization, sample absorption, and Compton scattering were applied to the diffraction patterns to obtain the structure function $S(Q)$. Direct Fourier transformation of the reduced structure function $F(Q)=Q[S(Q)-1]$ up to $Q_{\max }$ $\approx 18 \AA^{-1}$ gave the PDFs $G(r)$. Here $G(r)=4 \pi r\left[\rho(r)-\rho_{\mathrm{o}}\right]$, where $\rho(r)$ and $\rho_{\mathrm{o}}$ are the instantaneous and average densities and $r$ is the radial distance between atom pairs. The extraction of $G(r)$ and the structural refinement were conducted within the analysis software PDFFIT [15].
Particular care was required to quantitatively fit a structural model to the experimental PDF data, due to the complexity of the $\alpha$-cubic crystalline structure $(P m \overline{3}, a \approx 12.5 \AA$, 11 Wyckoff positions (i.e. unique atom sites) and 138 atoms per unit cell) compared with structures typically refined using PDF methods. It proved useful to compare the fit of the crystalline $\alpha$-cubic phase model with the PDFs for each sample applying crystallographic symmetry constraints. While unconstrained refinement may yield a model that appears to better fit the data, this is unlikely to offer a unique solution and does not necessarily provide a reliable representation of the structure. Refinement of the structural model against the PDF data for $\alpha$-cubic $\mathrm{Al}-\mathrm{Fe}-\mathrm{Si}$ (Fig. 4a), refining the lattice parameter $(a=12.55 \AA)$, atomic displacement parameters, and atomic positions within the symmetry constraints, yielded a reliable fit (with a weighted profile R-factor, $R_{\mathrm{wp}}=13.9 \%$ ) [16]. The quality of this preliminary fit is comparable to reported refinements of single crystal diffraction data, although the weighting of the PDF by a pre-factor $Q$ (i.e. the Fourier transform of $F(Q)=Q[S(Q)-1]$ ), placing an emphasis on high $Q$ data, causes the numerical value of $R_{\mathrm{wp}}$ to be inflated relative to a Bragg refinement of equal quality. As crystalline Al may be within the scattering volume we attempted to fit the experimental data with $\alpha$-phase plus crystalline $\mathrm{Al}$, but we found no significant $\mathrm{Al}$ inclusions in this sample. For comparison the $\alpha$-cubic $\mathrm{Al}-\mathrm{Fe}-\mathrm{Si}$ model (Fig. 4b) refined against data from the ico-Al-Fe-Si phase yielded a less good fit ( $R_{\mathrm{wp}}=28.5 \%, a=12.06 \AA$ ).

Recent attempts to model the PDF of nanoparticle samples with uniform size distributions have applied damping 

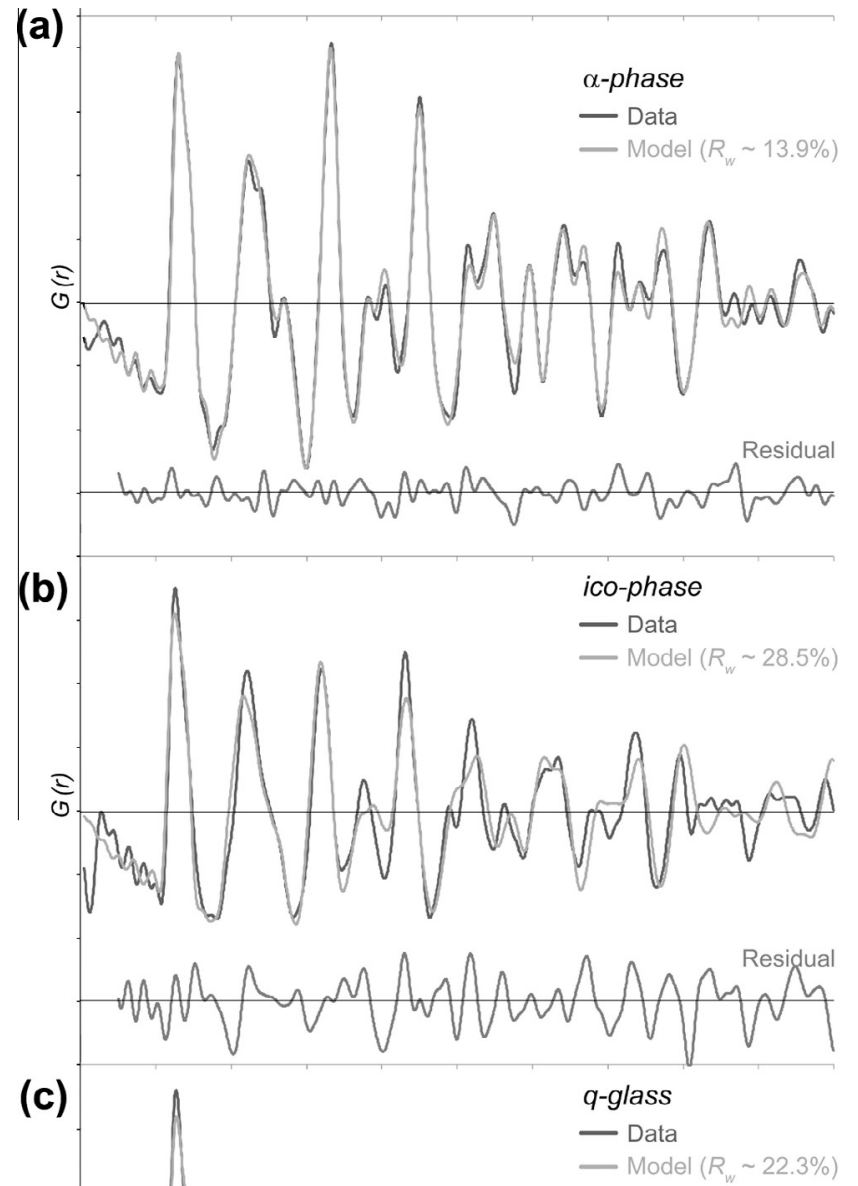

parameter $a=12.26 \AA$. This indicates a strong similarity between the local structure of the $\alpha-\mathrm{Al}-\mathrm{Fe}-\mathrm{Si}$ sample and the q-glass, indicative of the similarity between the local structures of the two samples. The q-glass structure fits less well to the neighboring icosahedral phase and not at all well to an Al-rich $\beta$-phase, as will be discussed later. Fig. 5a shows the $S(q)$ values of the three phases, offset vertically for clarity. Fig. 5b shows the total PDF $G(r)$ of the three phases overlaid for comparison and Fig. 5c displays $G(r)$ for the $\alpha$ - and the $\beta$-phases. The data in Fig. $5 b$ have been scaled (by 1.024r) for the q-glass and (by 1.041r) for the icosahedral phase to improve the overlay of the general features of the PDFs and to account for the compositiondependent mean atomic distances. For PDF analysis these distances are referred to as the refinement lattice parameters, shown in Table 1, of the different phases. The data in Fig. 5c cannot be scaled, as the two phases are unrelated.

The first correlation, evident at $\sim 2.7 \AA$, has slight asymmetry and increased width relative to that associated with the Debye temperature alone. Such asymmetry usually indicates that this peak contains contributions from different first shell correlations centered about this distance. From 4 to $6 \AA$ more significant differences in the PDFs become apparent. In both the $\alpha$-cubic $\mathrm{Al}-\mathrm{Fe}-\mathrm{Si}$ and the g-Al-Fe-Si phases a correlation at $4.7 \AA$ is resolved from a less intense feature at $5.7 \AA$. In the ico-Al-Fe-Si phase there is a single prominent feature at $4.7 \AA$ with a slight shoulder at higher $r$. We deduce from the partial pair correlations in the cubic $\alpha-\mathrm{Al}-\mathrm{Fe}-\mathrm{Si}$ phase (see Fig. 6) that this feature is due to an $\mathrm{Al}-\mathrm{Al}$ correlation. At $8 \AA$ there is a double peak structure for $\alpha-\mathrm{Al}-\mathrm{Fe}-\mathrm{Si}$ and $\mathrm{g}-\mathrm{Al}-\mathrm{Fe}-\mathrm{Si}$, but only a single peak for ico-Al-Fe-Si.

The peaks in the PDF for the crystalline $\alpha$-cubic phase and for the icosahedral phase continue to high $r$, consistent with long-range order. In contrast, the intensity of the atom-atom correlations in the g-Al-Fe-Si phase PDF decrease with increasing $r$, with no well-defined features beyond $\sim 2 \AA$. This damping is consistent with the absence of long-range translational and rotational symmetry in a glassy material, although the peaks in this case persist to significantly longer length scales than for typical oxidebased or metallic glasses [18]. We note that $G(r)$ for from the q-glass decreases in amplitude around $12 \AA$, which indicates that the size of the structural units comprising the glass are comparable in size to the icosahedral clusters in the $\alpha$-cubic phase.

\section{Heat treatment and phase changes in the solid state}

Having determined the local structural ordering in the q-glass, we now turn to differentiating between a glass and a nano- or microcrystalline structure $[19,20]$. We join Chen and Spaepen in defining a microcrystalline solid as a material in which the grain structure coarsens upon annealing with no phase transition involved. To measure the phase development and their volume fractions we monitored the presence of diffraction peaks, linking directly to 

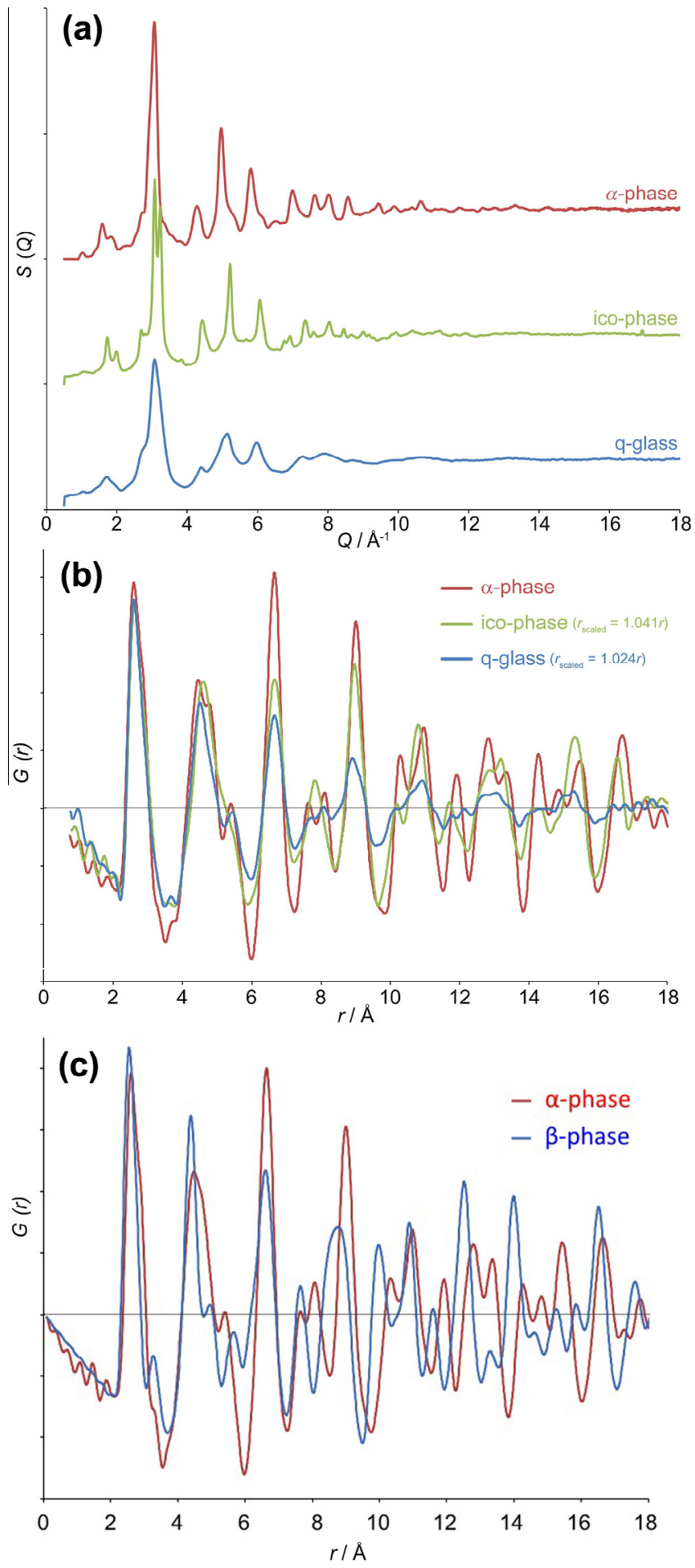

Fig. 5. (a) $S(Q)$ of the $\alpha$-phase, icosahedral phase and glass phase, offset vertically for clarity. (b) $G(r)$ of the $\alpha$-phase, icosahedral phase and glass phase overlaid for comparison. To improve the overlay of the general features of the PDFs and to account for the different lattice parameters of the different phases the data have been scaled (by 1.024r) for the glass and (by 1.041r) for the icosahedral phase. (c) $G(r)$ of the $\alpha$-phase and the $\beta$ phases overlaid for comparison.

the International Union of Crystallography definition of crystallinity [21]. Isothermal anneals of nano- or microcrystalline structures would show a simple narrowing of the

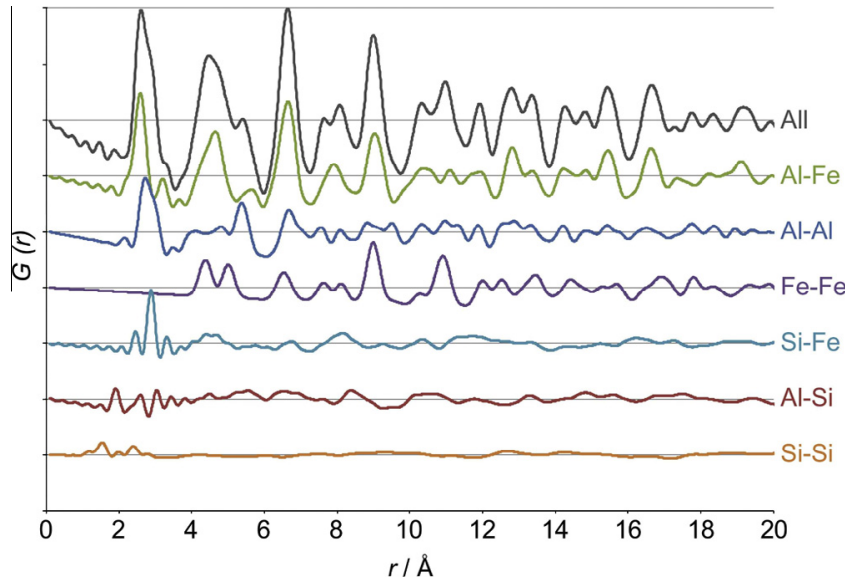

Fig. 6. Partial pair distributions of the six possible interatomic pairings in $\alpha$-cubic $\mathrm{Al}-\mathrm{Fe}-\mathrm{Si}$. The topmost shows a sum of all of the pairings. The known partial pair distributions within the $\alpha$-cubic $\mathrm{Al}-\mathrm{Fe}-\mathrm{Si}$ phase enables the comparison of the total $G(r)$ of this phase with the total $G(r)$ of $\mathrm{g}-\mathrm{Al}-\mathrm{Fe}-\mathrm{Si}$ to serve as a guide to the likely atomic pairings in the q-glass.

broadened diffraction peaks toward sharp Bragg peaks as the grains coarsen. A structure that is not crystalline will show evidence of a phase transformation, a period of coexistence with crystalline diffraction peaks growing as the residual broad glass peaks lose intensity without narrowing as the growing crystals consume the glass.

Appropriate temperatures for the in situ isothermal Xray measurements were identified by conducting preliminary isothermal calorimetric measurements on the q-glass at $320^{\circ} \mathrm{C}, 330^{\circ} \mathrm{C}$, and $335^{\circ} \mathrm{C}$. To monitor the series of structural transitions during annealing high energy $(\sim 58 \mathrm{keV}, \lambda \approx 0.213 \AA) \mathrm{X}$-ray scattering data suitable for PDF analysis were collected in beamline 11-ID-B at the Advanced Photon Source, Argonne National Laboratory. The sample was mounted orthogonal to a $200 \times 200 \mu \mathrm{m}$ X-ray beam within a temperature controlled sample environment (DSC Thermatica System) from Linkam Scientific Instruments. Data were collected at ambient temperature and during annealing of the q-glass at $330{ }^{\circ} \mathrm{C}(3 \mathrm{~h})$ and $305^{\circ} \mathrm{C}(16 \mathrm{~h})$. Data were collected during $1 \mathrm{~min}$ exposures at approximately $2 \mathrm{~min}$ intervals in the case of the short anneal, beginning immediately after a rapid ramp in temperature to $330^{\circ} \mathrm{C}$. Data were collected during 5 min exposures at approximately $6 \mathrm{~min}$ intervals during the $16 \mathrm{~h}$ isothermal hold at $305^{\circ} \mathrm{C}$, after which there was an additional rapid ramp to $345^{\circ} \mathrm{C}$, where we took the last $4 \mathrm{~h}$ of isothermal data. Every tenth image was dedicated to measuring the dark field. Additional data were collected upon recovery of the annealed sample, with greater than $2 \theta$ resolution (longer sample to detector distance), to assist in phase identification.

For each of the three preliminary isothermal measurements a first-order phase transition was observed. The phases(s) present during this transformation were identified from the in situ synchrotron XRD data obtained during the $330{ }^{\circ} \mathrm{C}$ isothermal anneal as described above. We found that the q-glass persisted for about $40 \mathrm{~min}$, after which it 
was transformed via a first-order phase transition to $\beta-\mathrm{Al}_{4.5} \mathrm{FeSi}[22]$. The $\beta-\mathrm{Al}_{4.5} \mathrm{FeSi}$ structure is a monoclinic pseudo-tetragonal double layering of double-capped (A1,Si) square antiprisms with shared edges, with periodic stacking faults along the $c$-axis. The 10 -coordinated $\mathrm{Fe}$ atoms lie at the center of the antiprisms. The PDF results shown in Fig. 5c highlight the differences between this phase and the $\alpha$-phase.

During an extended isothermal anneal at $305^{\circ} \mathrm{C}$ the $\mathrm{q}-$ glass exhibited more complex behavior. Fig. 7 shows the appearance of reflections indicative of fcc Al in the early in situ data. Since we required a $200 \times 200 \mu \mathrm{m}$ X-ray beam for in situ annealing data collection, compared with the $10 \times 10 \mu \mathrm{m}$ X-ray beam for static data collection, the presence of fine scale Al texture could not be entirely avoided in the q-glass data. By following the growth of the intensity of the crystalline $\mathrm{Al}$ diffraction peaks as a function of time we discovered that q-glass rejects excess $\mathrm{Al}$, producing fcc $\mathrm{Al}$. This is the solid-solid equivalent of the solid-liquid process during rapid solidification, and indicates that the glass has solubility limits that are temperature dependent. After the q-glass rejected $\mathrm{Al}$ this two-phase mixture persisted for approximately $1 \mathrm{~h}$ until a new phase, again $\beta-\mathrm{Al}_{4.5} \mathrm{FeSi}$, grew in, consuming both the remaining q-glass and most, but not all, of the fcc Al. This is a classical peritectoid reaction in which two phases, in our case the fcc Al and the q-glass, react to form another phase, $\beta-\mathrm{Al}_{4.5} \mathrm{FeSi}$. We see in Fig. 8a, where the crystalline $\mathrm{Al}$ and $\beta-\mathrm{Al}_{4.5} \mathrm{FeSi}$ peak intensities are plotted as a function of time, that after this peritectoid phase transformation is complete there is another stable period during which polycrystalline $\mathrm{Al}$ and $\beta-\mathrm{Al}_{4.5} \mathrm{FeSi}$ coexist. Analyses of the product by means of PDF methods, where the scans from the long stable period

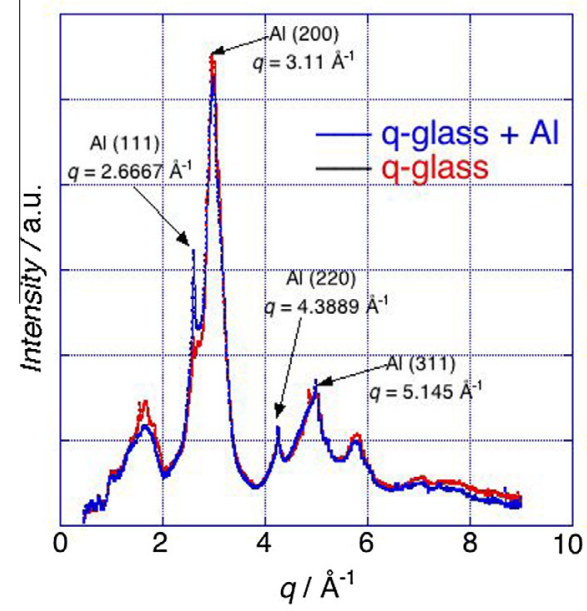

Fig. 7. Diffraction from g-Al-Fe-Si (red) and g-Al-Fe-Si with $\mathrm{Al}$ inclusions (blue). Note that some of the $\mathrm{Al}$ diffraction peaks fall on the broad $\mathrm{g}-\mathrm{Al}-\mathrm{Fe}-\mathrm{Si}$ rings. A small amount of nano-crystalline $\mathrm{Al}$ texture is evident in the q-glass measurement due to the larger slit size required for the isothermal annealing measurements. (For interpretation of the references to color in this figure legend, the reader is referred to the web version of this article.)
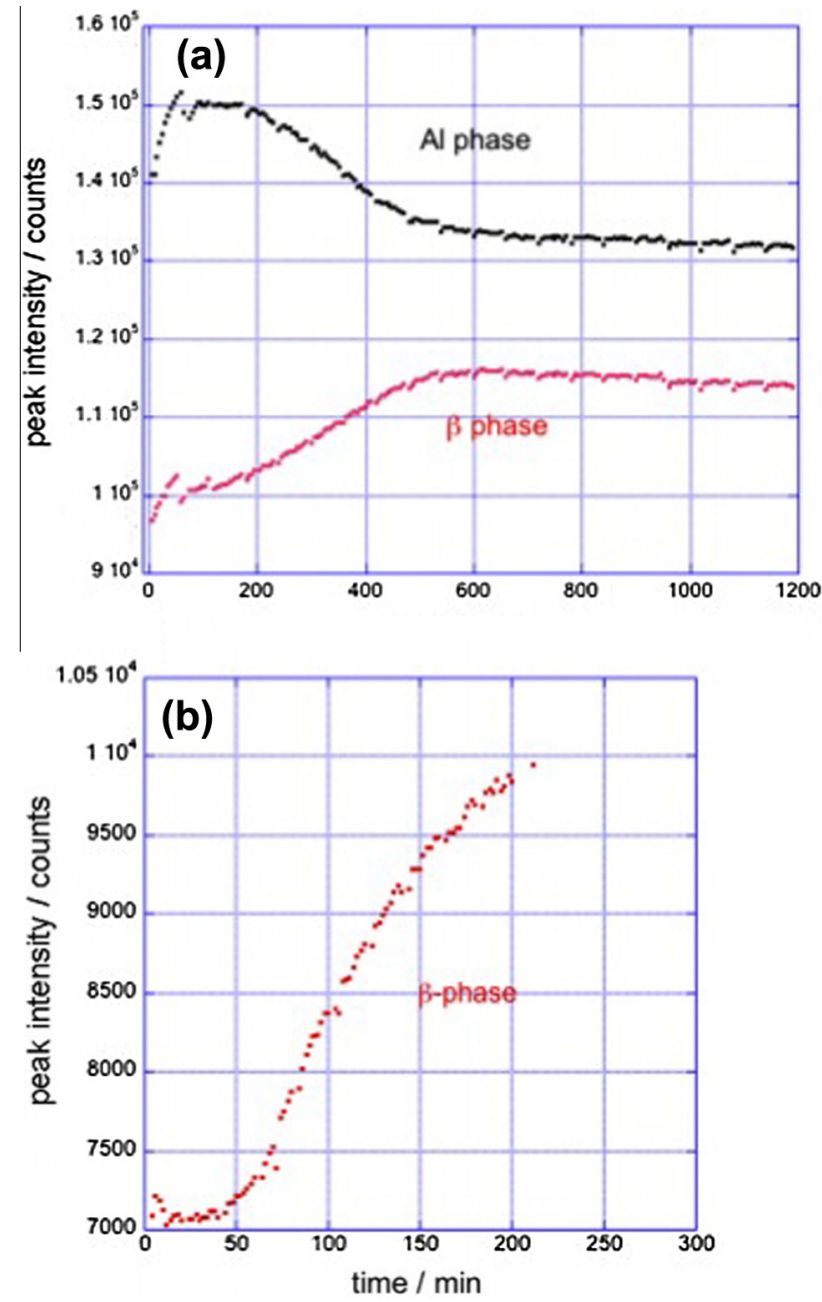

Fig. 8. Peak intensities for the crystalline phases as a function of time during the (a) $305^{\circ} \mathrm{C}$ and (b) $330^{\circ} \mathrm{C}$ thermal anneals show the increase in $\beta$-phase and increase and later decrease in the fcc $\mathrm{Al}$ phase at $305^{\circ} \mathrm{C}$. As a result of a declining X-ray beam intensity the plateau that start at $600 \mathrm{~min}$ are actually flat and indicate that equilibrium has been reached.

are summed, and Rietveld refinement of a higher resolution diffraction pattern, agree to within $1 \%$ that the product is 90 wt. $\% \beta_{-}-\mathrm{Al}_{4.5} \mathrm{FeSi}$ and $10 \mathrm{wt} . \%$ fcc $\mathrm{Al}$ (Fig. 9). Clearly, the fcc Al is not entirely consumed, and furthermore no additional phases are formed. We next subtracted the Al to derive the structure of the "relaxed" glass. The result is shown in Fig. 10, where we show the structure of the q-glass before the isothermal anneal compared with that of the in situ relaxed q-glass during isothermal annealing. The two structures are nearly identical.

Since there is no evidence in the $330{ }^{\circ} \mathrm{C}$ data for the formation of fcc $\mathrm{Al}$ (Fig. 8b) and there is no indication of a transformation to a more stable q-glass, we conclude that the q-glass has a larger compositional stability range at the higher temperature. However, even when there is no evolution of the q-glass to a relaxed structure, as during the $305^{\circ} \mathrm{C}$ anneal, the product is the same as that of the higher temperature anneal, namely $\beta-\mathrm{Al}_{4.5} \mathrm{FeSi}$, which is quite different from the structure of the original q-glass. 

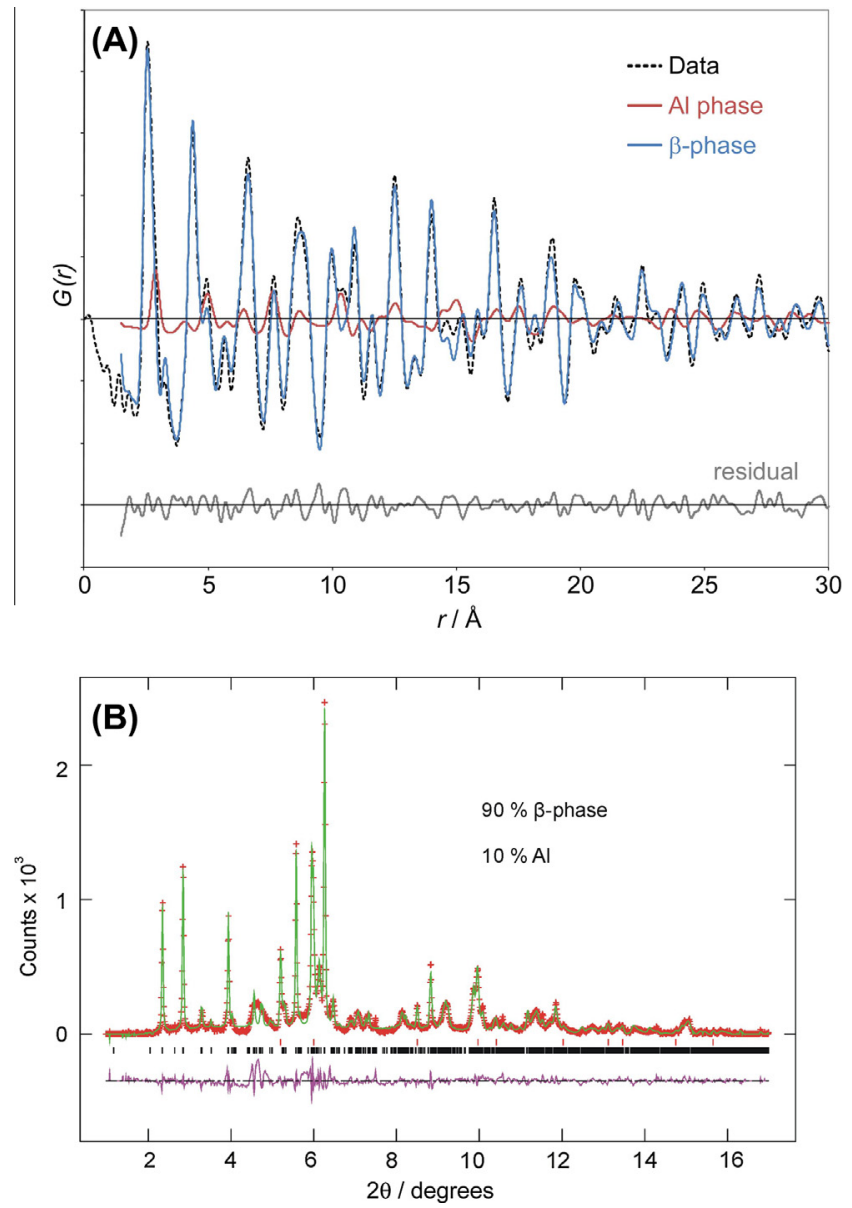

Fig. 9. Analysis of the product of the $305^{\circ} \mathrm{C}$ anneal. (A) Model of the summed PDF data from 600 to $1200 \mathrm{~min}$, indicating an excellent fit to 11 wt. $\% \mathrm{Al}$ and 89 wt. $\% \beta-\mathrm{Al}_{4.5} \mathrm{FeSi}$. (B) Rietveld refinement of XRD results for the product of the $305^{\circ} \mathrm{C}$ anneal. Tick marks indicate calculated peak positions for $\beta$-phase (lower marks) and $\mathrm{Al}$ (upper marks). $\lambda=0.2128 \AA$. The analysis finds $90 \mathrm{wt} . \% \beta-\mathrm{Al}_{4.5} \mathrm{FeSi}$ and $10 \mathrm{wt} . \%$ crystalline $\mathrm{Al}$. The data prove that $\beta-\mathrm{Al}_{4.5} \mathrm{FeSi}$ and $\mathrm{Al}$ remain at the end of anneal.

\section{Discussion and conclusions}

The q-glass is an isotropic solid obtained by rapid cooling of a melt. It forms by nucleation and growth along a moving interface between solid and melt with partitioning of the chemical elements, thereby proving that this solid is not a frozen melt. These features appear in the micrograph of an Al-rich specimen [2], where we see the formation of a two phase lamellar composite that is a eutectic solid composed of fcc Al and q-glass. We obtained some evidence for partitioning from an energy dispersive X-ray spectroscopy (EDS) analysis of the compositions of the isotropic solid and the eutectic composite. Additional evidence of partitioning comes from the narrow $(\sim 10 \mathrm{~nm})$ white band around each nodule. This is a diffusion zone rich in rejected aluminum with a thickness that is approximately $D / v$, where $D$ is a diffusion coefficient and $v$ is the nodule interface growth velocity. Assuming a typical liquid phase diffusion constant of $D \approx 10^{-9} \mathrm{~m}^{2} \mathrm{~s}_{\wedge}^{-1}$ implies that

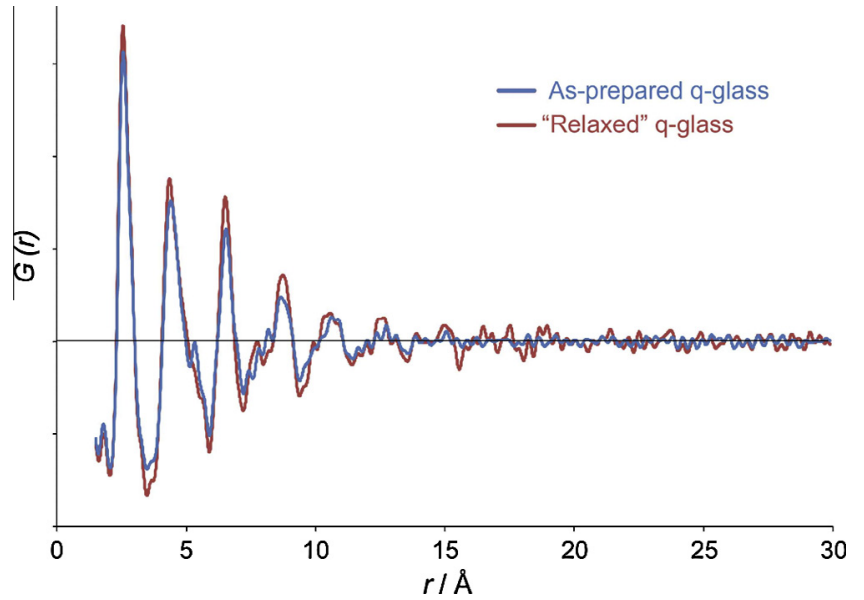

Fig. 10. The as-prepared q-glass $G(r)$ was derived from an ex situ measurement before the $305^{\circ} \mathrm{C}$ thermal anneal, where crystalline $\mathrm{Al}$ was not subtracted from these data. The annealed "relaxed" q-glass result was derived by subtracting $\mathrm{Al}$ from the summed scans measured during the plateau in $\mathrm{Al}$ intensity at $100-160 \mathrm{~min}$ during the in situ $305^{\circ} \mathrm{C}$ thermal anneal. In this case $G(r)$ above $r=15 \AA$ was fitted to crystalline Al, and the result was subtracted from the total $G(r)$ to give the relaxed q-glass component.

$v \approx 0.1 \mathrm{~m} \mathrm{~s}_{\curlywedge}^{-1}$. As the nodules surrounded by such diffusion zones grow they become unstable [23], where the onset of this instability is seen in the undulations in the interface that provide additional evidence for partitioning. The transverse section in Fig. 1b provides evidence that the nearly $100 \%$ q-glass samples also form by nucleation and growth.

An alternative explanation for the observed separation into two components is phase separation into two liquids of different composition that, on further cooling, become the q-glass and a eutectic. However, we eliminated this possibility in earlier experiments by varying the composition of the alloy melt and observing that as the overall Al content was reduced the isotropic solid continued to be the first phase to form and eventually became the major phase $[2,4]$. This ruled out liquid phase separation.

It is always possible to find local atomic arrangements in liquids and glasses that match pieces of crystals and even of quasi-crystals. Whether such pieces are tiny crystals and the glass is a polycrystalline aggregate cannot be decided from structural data. We define a microcrystalline solid as a material in which the grain structure coarsens upon annealing with no phase transition involved. During in situ annealing we found instead that the broad XRD rings from the q-glass remained broad and gradually diminished in intensity, while sharp diffraction peaks from unrelated crystalline phases appeared and grew during the anneal. This is consistent with a first order phase transition.

During the $305^{\circ} \mathrm{C}$ anneal the q-glass persisted for more than $1 \mathrm{~h}$ before gradual nucleation and growth of a $\beta$-phase. This demonstrated that the q-glass is a metastable phase in the classical sense, i.e. stable to small perturbations and unstable only to the appearance of $\beta$-phase 
"nuclei" particles that are large enough to overcome capillary barriers from the interfaces that have to be created.

The q-glass has strong local chemical ordering, which is common in intermetallic crystalline phases of the same three elements. This ordering should depend upon composition and temperature. We looked for, but could not find, diffraction evidence of relaxation of the q-glass structure (Fig. 10). At $305^{\circ} \mathrm{C}$ we observed a steady growth of fcc Al peaks in the diffraction pattern (Fig. 8a) as Al is rejected by the q-glass. As expected for a two phase equilibrium the rate of fcc $\mathrm{Al}$ phase formation slows until it is almost undetectable as metastable equilibrium is approached.

During the $330{ }^{\circ} \mathrm{C}$ anneal (Fig. $8 \mathrm{~b}$ ) the q-glass persisted for $\sim 40$ min before gradual nucleation and growth of the $\beta$-phase. During this anneal there was no diffraction evidence of $\mathrm{Al}$ rejection. We conclude that the solubility of aluminum in the q-glass is broad enough at this temperature to incorporate the $\mathrm{Al}$ present.

Eventually annealing at both temperatures resulted in the nucleation and growth of a distinct $\beta$-phase that consumed the q-glass. Because of the symmetry change and the lack of structural similarity to the original motifs within the q-glass this is also a first order phase transition. Because the q-glass is composed of three elements it can coexist at constant pressure with up to two other phases over a range of temperatures. During growth of the $\beta$-phase at $305^{\circ} \mathrm{C}$ the q-glass coexisted with both the $\beta$-phase and the rejected fcc Al. This difference between the two anneals indicates that the solvus of the $\beta$-phase for aluminum is also temperature dependent.

Like all thermodynamic phases, the q-glass can participate in heterogeneous coexistence with other phases along interfaces where, away from critical points, the composition and structure change over atomic scale distances. In general such interfaces move to reduce the free energy as phases expand or shrink, compositions adjust, and the area of interfaces changes. The presence of interfaces establishes that the adjoining regions are of different phase. If adjoining regions were of the same phase there would be no interface, only a spreading diffusion zone. We have identified three phases that form interfaces with the q-glass. During formation upon rapid cooling the q-glass forms interfaces with the initial melt and with fcc $\mathrm{Al}$ in eutectic structures. During an isothermal anneal the glass forms interfaces with fcc Al precipitates and the $\beta$-phase. These last two are also possible with a glass that is a frozen melt, but the coexisting compositions of these phases in the q-glass are different from what is expected with a frozen melt.

We have demonstrated that the q-glass is a solid solution phase in the thermodynamic sense. It is neither a frozen melt nor nanocrystalline. The large ordering distance of $\sim 12 \AA$ shown in the PDF results in Fig. 5b demonstrates that a large fraction of the atoms maintain a well-defined positional relationship that, within this range, is consistent with an ordered solid. Nevertheless, the phase is unquestionably homogeneously disordered, e.g. with no preferred growth or structural directionality. To our knowledge this combination of traits has never before been reported, hence the impetus to understand the underlying structural properties of this unique phase.

The existence of a growth front with the melt implies that the atomic surface of the q-glass forms the template for successive growth. We need to understand why, during growth of the q-glass, the directional order that typifies crystals and quasi-crystals is lost. This behavior must also be reconciled with the high degree of local ordering demonstrated by the PDF results.

The PDF data indicate that the local structure of the glass closely resembles that of the $\alpha$-cubic $\mathrm{Al}-\mathrm{Fe}-\mathrm{Si}$ phase, which is composed of concentric shell Mackay icosahedra that maintain their orientation by packing along specific 3 -fold icosahedral directions [23]. Similar clusters are found with a variety of different packing configurations [24] in other systems. Additional evidence of unexpected directional order of these structural units has been observed in TEM studies of polycrystalline aggregates. Although the axes of adjacent crystals differ by 5 -fold rotations the structural units maintain the same orientation [26].

One possible explanation for the loss of orientational order is the presence of local frustration during growth. If incompatible packing configurations grow from the same seed cluster local frustration would likely disrupt the longrange ordering. Such disordered packings of Mackaybased clusters in two dimensions have been observed using TEM in the Ti-Mn-Si system [27].

A more intriguing possibility is that the q-glass is a fully ordered atomic structure that possesses no long-range translational symmetry and is isotropic. This too is consistent with the PDF results. As mentioned in the Introduction, there are many 3-D configurations of points that exhibit these properties [1]. The aperiodic zoo discussed in the Appendix describes some 2-D examples. The Appendix also offers additional theoretical considerations regarding fully ordered isotropic structures. The quasi-crystalline phases taught us that perfect atomic ordering does not require translational symmetry. If the q-glass turns out to be fully ordered then the requirement for finite rotational symmetry in an ordered solid would also prove incorrect.

We know of no experimental measurement that can distinguish between these two models. Diffraction is a standard probe of atomic structure and the presence of sharp diffraction peaks proves the existence of long-range ordering. However, we are faced with the possibility that the reverse may not be true, that glass-like scattering may result from highly ordered atomic arrangements.

\section{Disclaimer}

Certain commercial instruments are identified in this paper to foster understanding. Such identification does not imply recommendation or endorsement by the Department of Commerce or the National Institute of Standards and Technology, nor does it imply that the equipments identified are necessarily the best available for the purpose. 


\section{Uncited reference} $[25]$.

\section{Acknowledgements}

The authors acknowledge Frank Biancaniello for alloy preparation and Alexander Shapiro for assistance with the SEM measurements. We also thank Maureen Williams for X-ray characterization and Kil-Won Moon for isothermal calorimetry. We thank Marjorie Senechal for useful discussions. The X-ray studies were conducted in beamlines 1-ID, 11-ID and 32-ID at the Advanced Photon Source (APS). This research would not have been possible without the unique capabilities of the dedicated pair distribution function X-ray scattering instrument at 11-ID-B at the APS. This instrument provides data over a broad range in Fourier space, offering the necessary high spatial resolution at intermediate length scales (6-20 $\mathrm{A}$ ). It is adequately sensitive to probe very small sample volumes $\left(10^{3} \mu \mathrm{m}^{3}\right)$ and still provide adequate counting statistics to perform in situ real time measurements of phase transformations. The APS is an Office of Science User Facility operated for the US Department of Energy (DOE) Office of Science by Argonne National Laboratory, and was supported by the DOE under contract no. DE-AC02-06CH11357.

\section{Appendix A}

\section{A.1. The aperiodic zoo}

The International Union of Crystallography definition of crystals is based on the existence of sharp diffraction peaks and includes both crystals and quasi-crystals. It would also include layered structures such as pyrolytic graphite that are stacked periodically, even though the layers are randomly oriented, while most 2-D and 3-D isotropic structures would be excluded. Within the limit the pinwheel tiling described by Radin [28] includes all orientations and is an example of an ordered 2-D arrangement of points that is neither periodic nor quasi-periodic, and even within the limit is not isotropic. The quasi-periodic 2-D tiling with a 19-fold axis shown in Fig. A1A approximates an isotropic structure and its diffraction pattern in Fig. A1B shows glass-like rings, although it is composed of Bragg peaks. We believe that within the limit of infinite rotations the diffraction from such a structure would approximate that from a 2-D glass. Techniques other than diffraction would be required to establish the degree of ordering in such a system.

Computer simulations have identified transitions from liquids to ordered isotropic phases. In 1992 Dzugutov [29] proposed an artificial potential for a single component system in which non-crystalline structures would have lower energies than crystalline structures. Simulations found a first order reversible phase transition between a melt and a non-crystalline isotropic solid that is highly structured and has low entropy. Mendelev [30] found a similar first-order reversible transition in a molecular dynamics simulation that used an early embedded atom potential for $\mathrm{Al}$. He found a melting temperature where the two phases coexist along an interface. Above this temperature melting of the solid occurred heterogeneously by motion of the interface, and not by homogeneous processes throughout the solid. Below the melting temperature the interface moved into the melt. Mendelev [30] reported a latent heat, a volume change and a $10^{4}$ change in diffusivity. Thus our observation that an isotropic solid phase can grow from the melt has some theoretical basis. These simulation studies prove that this can occur with some atomic potential. Our observations prove that it can occur in real systems.

\section{A.2. Fundamental theoretical considerations}

For a three component melt there are generally several temperatures at which solids are in equilibrium with melts. At the liquidus temperature a small amount of solid can coexist with the melt. Generally this will be a solid with a composition different from the melt and its growth will
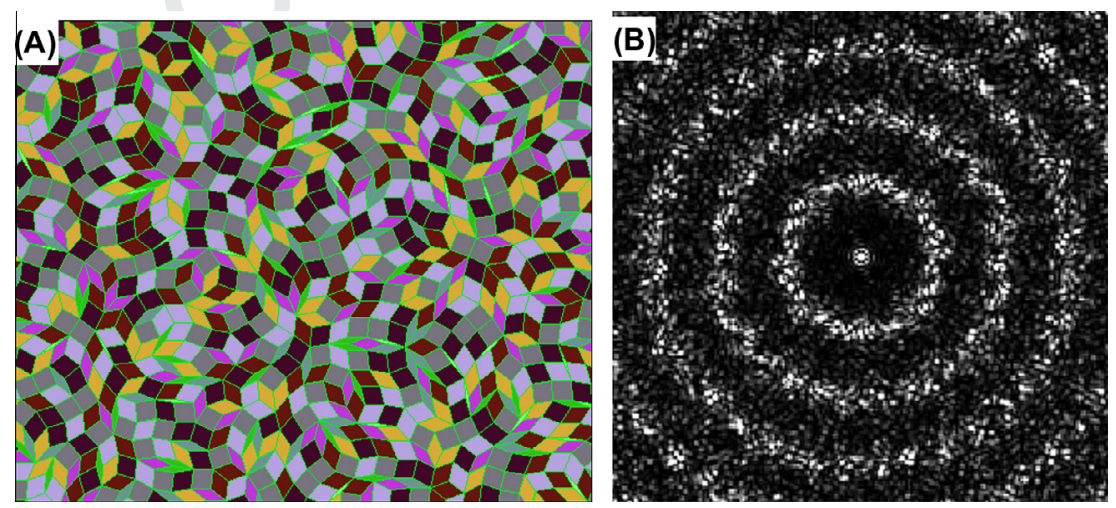

Fig. A1. (A) The quasi-periodic 2-D tiling with a 19-fold axis approximates an isotropic structure. (B) Its diffraction pattern shows glass-like rings, although it is composed of Bragg peaks. In the limit of infinite rotations the diffraction from such a structure would approximate that from a 2-D glass. (Calculated results from Steffen Weber's website at http://jcrystal.com/steffenweber/JAVA/jtiling/jtiling.html.) 
change the composition of the remaining melt. At and below the solidus temperature the melt has been fully replaced by solids. Generally there will be a mixture of solids, unless there is a solid with the same composition as that of the melt.

The liquidus and solidus temperatures coincide for special compositions. "Congruent" compositions occur where a melt and a single solid of the same composition are in equilibrium at a congruent temperature, usually at temperature maxima. Eutectic compositions occur when at the liquidus a melt can decompose fully into a mixture of two or more solids. Eutectic temperatures are at minima. Of special interest for this research is the case when a melt that is not of a eutectic composition encounters a liquidus on cooling. The growth of that first phase will change the melt composition, sometimes towards a eutectic. This is what is seen in Fig. 1. The q-glass nodules are the first phase to form. They differ in composition from the melt, and reject aluminum into it. When the melt reaches the eutectic composition two phases, whose volume-averaged composition matches that of the remaining melt, grow outward from the nodules.

A microcrystalline model of metallic glasses has been proposed by one us (L.B.) based on an experimental study of how grain size depends on cooling rate. Isotropic featureless specimens were found where the grain size approached the atomic scale, raising the question of whether these were metallic glasses or polycrystalline aggregates with a grain size of less than $1 \mathrm{~nm}$. Their energy would exceed that of large crystals by the presence of $\sim 10^{9} \mathrm{~m}^{2}$ grain boundaries, having an energy of $\sim 10^{8} \mathrm{~J} \mathrm{~m}^{-3}$ specimen, and comparable with the heat of fusion. We suggest that it is unreasonable to assume a model where the crystals have sizes close to or even smaller than the unit cells. Some claim even to have distinguished between microcrystals and quasi-microcrystals on this scale. If the specimen is indeed microcrystalline no further phase change is needed for coarsening of the grains on heating to sizes where their crystallinity is not in doubt. Such a process can be observed in XRD spectra as broad glassy peaks narrow and become resolved as individual crystalline peaks.

\section{References}

[1] Senechal M. In: Quasicrystals and geometry. New York: Cambridge University Press; 1995 [chapter 7].

[2] Long GG, Chapman KW, Chupas PJ, Bendersky LA, Levine LE, Mompiou F, et al. Phys Rev Lett 2013;111:015502.

[3] Bendersky LA, Biancaniello FS, Schaefer RJ. J Mater Res 1987:2:427.

[4] Cahn JW, Bendersky LA. Mater Res Soc Symp Proc 2004;806:MM2.7.1.

[5] Bendersky LA, Kaufman MJ, Boettinger WJ, Biancaniello FS. Mater Sci Eng 1988;98:213.

[6] Cooper M. Acta Cryst 1967;23:1106.

[7] Sugiyama K, Kaji N, Hiraga K. Acta Cryst 1998;C54:445.

[8] Ghosh G. Aluminum-iron-silicon, ternary alloys, vol. 5. Weinheim: Wiley-VCH; 1992. p. 394.

[9] Liu Z-K, Chang YA. Metall Mater Trans 1999;A30:1081.

[10] Toby BH, Egami T. Acta Cryst 1992;A48:336.

[11] Shastri SD, Fezzaa K, Mashayeki A, Lee W-K, Fernandez PB, Lee PL. J Synch Rad 2002;9:317.

[12] Chupas PJ, Chapman KW, Lee PL. J Appl Cryst 2007:40:463.

[13] Hammersley AP. ESRF98HA01T, FIT2D V9.129 Reference Manual V3.1. ?: ?, 1998.

[14] Hammersley AP, Svensson SO, Thompson A, Grafsma H, Kvick Å, Moy JP. Rev Sci Instrum 1995;66:2729.

[15] Proffen T, Billinge SJL. J Appl Cryst 1999;32:572.

[16] Toby BF. Powder Diffract 2006;21:67.

[17] Page K, Proffen T, Terrones H, Terrones M, Lee L, Yang Y, et al. Chem Phys Lett 2004;393:385.

[18] Ahn K, Louca D, Poon SJ, Shiflet GJ. J Phys Cond Mater 2003;15:A2357.

[19] Chen LC, Spaepen F. Mater Sci Eng 1991;A133:342.

[20] Chen LC, Spaepen F. Nature 1988;336:366.

[21] The Executive Committee of ? Acta Cryst 1992;A48:928.

[22] Hansen V, Hauback B, Sundberg M, Rømming C, Gjønnes J. Acta Cryst 1998;B54:351.

[23] Mullins W, Sekerka R. J Appl Phys 1963;34:323.

[24] Steurer W, Deloudi S. Crystallography of quasicrystals, concepts, methods and structures. Springer Series in Materials Sciences no. 126. Berlin: Springer Verlag; 2009. p. 243.

[25] Levine LE, Gibbons PC, Kelton KF. Phil Mag 1992;B65:435.

[26] Bendersky LA, Cahn JW, Gratias D. Phil Mag 1989;B60:837.

[27] Levine LE, Gibbons PC, Viano AM. Phil Mag 1994;B70:11.

[28] Radin C. Annals Math 1994;139:661.

[29] Dzugutov M. Phys Rev 1992;A46:R2984.

[30] Mendelev MI, Schmalian J, Wang CZ, Morris JR, Ho KM. Phys Rev 2006;B74:104206. 